\title{
MONOTONE ITERATIONS FOR DIFFERENTIAL EQUATIONS WITH A PARAMETER
}

\author{
TADEUSZ JANKOWSKI \\ Technical University of Gdansk, Department of Numerical Analysis \\ Gdansk, POLAND \\ V. LAKSHMIKANTHAM \\ Florida Institute of Technology, Applied Mathematics Program \\ Melbourne, FL 32901 USA
}

(Received February, 1997; Revised June, 1997)

Consider the problem

$$
\left\{\begin{array}{l}
y^{\prime}(t)=f(t, y(t), \lambda), \quad t \in J=[0, b] \\
y(0)=k_{0} \\
G(y, \lambda)=0
\end{array}\right.
$$

Employing the method of upper and lower solutions and the monotone iterative technique, existence of extremal solutions for the above equation are proved.

Key words: Monotone Iterations, Differential Equations, Monotone Iterative Technique.

AMS subject classifications: $34 \mathrm{~A} 45,34 \mathrm{~B} 99$.

\section{Preliminaries}

Consider the following differential equation

$$
x^{\prime}(t)=f(t, x(t), \lambda), \quad t \in J=[0, b]
$$

with the boundary conditions

$$
x(0)=k_{0}, \quad x(b)=k_{1},
$$

where $f \in C(J \times R \times R, R)$ and $k_{0}, k_{1} \in R$ are given. The corresponding solution of (1) yields a pair of $(x, \lambda) \in C^{1}(J, R) \times R$ for which problem (1) is satisfied. Problem (1) is called a problem with a parameter.

Conditions on $f$ which guarantee the existence of solutions to (1) are important analysis theorems. Such theorems can be formulated under the assumption that $f$ satisfies the Lipschitz condition with respect to the last two variables with suitable 
Lipschitz constants or Lipschitz functions [1-3, 5].

This paper applies the method of lower and upper solutions for proving existence results [4]. Using this technique, we construct monotone sequences, giving sufficient conditions under which they are convergent. Moreover, this method gives a problem solution in a closed set.

Note that $x(b)$ in condition $(1 b)$ may appear in a nonlinear way, so it is a reason that we consider the following problem in the place of (1):

$$
\left\{\begin{array}{l}
y^{\prime}(t)=f(t, y(t), \lambda), \quad t \in J=[0, b] \\
y(0)=k_{0} \\
G(y, \lambda)=0
\end{array}\right.
$$

where $f \in C(J \times R \times R, R), G \in C(R \times R, R)$.

\section{Main Results}

A pair $(v, \alpha) \in C^{1}(J, R) \times R$ is said to be a lower solution of (2) if:

$$
\left\{\begin{array}{l}
v^{\prime}(t) \leq f(t, v(t), \alpha), \quad t \in J \\
v(0) \leq k_{0} \\
0 \leq G(v, \alpha)
\end{array}\right.
$$

and an upper solution of (2) if the inequalities are reversed.

Theorem 1: Assume that $f \in C(J \times R \times R, R), G \in C(R \times R, R)$, and:

$1^{\circ} y_{0}, z_{0} \in C^{1}(J, R), \lambda_{0}, \gamma_{0} \in R$, such that $\left(y_{0}, \lambda_{0}\right),\left(z_{0}, \gamma_{0}\right)$ are lower and

upper solutions of problem (2) such that $y_{0}(t) \leq z_{0}(t), t \in J$ and, $\lambda_{0} \leq \gamma_{0}$;

$2^{\circ} \quad f$ is nondecreasing with respect to the last two variables;

$3^{\circ} \quad G$ is nondecreasing with respect to the first variable;

$4^{\circ} \quad G(y, \lambda)-G(y, \beta) \leq N(\beta-\lambda)$ for $y_{0}(t) \leq y(t) \leq z_{0}(t), \quad t \in J, \quad \lambda_{0} \leq \lambda \leq \beta$ $\leq \gamma_{0}$ with $N \geq 0$.

Then there exist monotone sequences $\left\{y_{n}, \lambda_{n}\right\},\left\{z_{n}, \gamma_{n}\right\}$ such that $y_{n}(t) \rightarrow y(t)$, $z_{n}(t) \rightarrow z(t), \quad t \in J ; \lambda_{n} \rightarrow \lambda, \gamma_{n} \rightarrow \gamma$ as $n \rightarrow \infty$; and this convergence is uniformly and monotonically on J. Moreover, $(y, \lambda),(z, \gamma)$ are minimal and maximal solutions of problem (2), respectively.

Proof: From the above assumptions, it is known that:

$$
\left\{\begin{array} { c } 
{ y _ { 0 } ^ { \prime } ( t ) \leq f ( t , y _ { 0 } ( t ) , \lambda _ { 0 } ) , \quad y _ { 0 } ( 0 ) \leq k _ { 0 } , } \\
{ 0 \leq G ( y _ { 0 } , \lambda _ { 0 } ) , }
\end{array} \quad \left\{\begin{array}{c}
z_{0}^{\prime}(t) \geq f\left(t, z_{0}(t), \gamma_{0}\right), \quad z_{0}(0) \geq k_{0} \\
0 \geq G\left(z_{0}, \gamma_{0}\right)
\end{array}\right.\right.
$$

and $y_{0}(t) \leq z_{0}(t), t \in J, \lambda_{0} \leq \gamma_{0}$. Let $\left(y_{1}, \lambda_{1}\right),\left(z_{1}, \gamma_{1}\right)$ be the solutions of:

and

$$
\left\{\begin{aligned}
y_{1}^{\prime}(t) & =f\left(t, y_{0}(t), \lambda_{0}\right), \quad y_{1}(0)=k_{0} \\
0 & =G\left(y_{0}, \lambda_{0}\right)-N\left(\lambda_{1}-\lambda_{0}\right)
\end{aligned}\right.
$$

$$
\left\{\begin{aligned}
z_{1}^{\prime}(t) & =f\left(t, z_{0}(t), \gamma_{0}\right), \quad z_{1}(0)=k_{0} \\
0 & =G\left(z_{0}, \gamma_{0}\right)-N\left(\gamma_{1}-\gamma_{0}\right)
\end{aligned}\right.
$$


respectively.

Put $p=\lambda_{0}-\lambda_{1}$, so:

$$
0=G\left(y_{0}, \lambda_{0}\right)-N\left(\lambda_{1}-\lambda_{0}\right) \geq-N\left(\lambda_{1}-\lambda_{0}\right)=N_{p},
$$

thus $p \leq 0$ and $\lambda_{0} \leq \lambda_{1}$. Now let $p=\lambda_{1}-\gamma_{1}$. In view of $3^{\circ}$ and $4^{\circ}$, we have:

$$
\begin{aligned}
0=G\left(y_{0}, \lambda_{0}\right) & -N\left(\lambda_{1}-\lambda_{0}\right)=G\left(y_{0}, \lambda_{0}\right)-G\left(z_{0}, \gamma_{0}\right)-N\left(\lambda_{1}-\lambda_{0}\right)+N\left(\gamma_{1}-\gamma_{0}\right) \\
& \leq G\left(z_{0}, \lambda_{0}\right)-G\left(z_{0}, \gamma_{0}\right)-N\left(\lambda_{1}-\lambda_{0}\right)+N\left(\gamma_{1}-\gamma_{0}\right) \\
& \leq N\left(\gamma_{0}-\lambda_{0}\right)-N\left(\lambda_{1}-\lambda_{0}\right)+N\left(\gamma_{1}-\gamma_{0}\right)=-N p .
\end{aligned}
$$

Hence $\lambda_{1} \leq \gamma_{1}$. Set $p=\gamma_{1}-\gamma_{0}$, so that:

$$
0=G\left(z_{0}, \gamma_{0}\right)-N\left(\gamma_{1}-\gamma_{0}\right) \leq-N\left(\gamma_{1}-\gamma_{0}\right)=-N p
$$

and thus $\gamma_{1} \leq \gamma_{0}$. As a result, we have:

$$
\lambda_{0} \leq \lambda_{1} \leq \gamma_{1} \leq \gamma_{0}
$$

We shall show that

$$
y_{0}(t) \leq y_{1}(t) \leq z_{1}(t) \leq z_{0}(t), \quad t \in J .
$$

Let $p(t)=y_{0}(t)-y_{1}(t), t \in J$, so:

$$
p^{\prime}(t)=y_{0}^{\prime}(t)-y_{1}^{\prime}(t) \leq f\left(t, y_{0}(t), \lambda_{0}\right)-f\left(t, y_{0}(t), \lambda_{0}\right)=0,
$$

and $p(0)=y_{0}(0)-y_{1}(0) \leq 0$. This shows that $p(t) \leq 0, t \in J$. Therefore $y_{0}(t) \leq$ $y_{1}(t), t \in J$. Put $p(t)=y_{1}(t)-z_{1}(t), t \in J$. In view of $2^{\circ}$, we have

$$
\begin{aligned}
p^{\prime}(t)= & y_{1}^{\prime}(t)-z_{1}^{\prime}(t)=f\left(t, y_{0}(t), \lambda_{0}\right)-f\left(t, z_{0}(t), \gamma_{0}\right) \\
& \leq f\left(t, z_{0}(t), \gamma_{0}\right)-f\left(t, z_{0}(t), \gamma_{0}\right)=0,
\end{aligned}
$$

and $p(0)=0$, so $p(t) \leq 0, t \in J$, and $y_{1}(t) \leq z_{1}(t), t \in J$. Put $p(t)=z_{1}(t)-z_{0}(t)$, $t \in J$. We obtain:

$$
p^{\prime}(t)=z_{1}^{\prime}(t)-z_{0}^{\prime}(t) \leq f\left(t, z_{0}(t), \gamma_{0}\right)-f\left(t, z_{0}(t), \gamma_{0}\right)=0,
$$

so $p(t) \leq 0, t \in J$, and hence $z_{1}(t) \leq z_{0}(t), t \in J$. This shows that (3) is satisfied.

Note that:

$$
y_{1}^{\prime}(t)-f\left(t, y_{0}(t), \lambda_{0}\right) \leq f\left(t, y_{1}(t), \lambda_{1}\right), y_{1}(0)=k_{0}
$$

and

$$
z_{1}^{\prime}(t)-f\left(t, z_{0}(t), \gamma_{0}\right) \geq f\left(t, z_{1}(t), \gamma_{1}\right), z_{1}(0)=k_{0} .
$$

Moreover, in view of $3^{\circ}$ and $4^{\circ}$, we have:

$$
\begin{gathered}
0=G\left(y_{0}, \lambda_{0}\right)-N\left(\lambda_{1}-\lambda_{0}\right) \leq G\left(y_{1}, \lambda_{0}\right)-N\left(\lambda_{1}-\lambda_{0}\right) \\
=G\left(y_{1}, \lambda_{0}\right)-G\left(y_{1}, \lambda_{1}\right)+G\left(y_{1}, \lambda_{1}\right)-N\left(\lambda_{1}-\lambda_{0}\right) \\
\leq N\left(\lambda_{1}-\lambda_{0}\right)+G\left(y_{1}, \lambda_{1}\right)-N\left(\lambda_{1}-\lambda_{0}\right)=G\left(y_{1}, \lambda_{1}\right),
\end{gathered}
$$

and

$$
\begin{aligned}
0 & =G\left(z_{0}, \gamma_{0}\right)-N\left(\gamma_{1}-\gamma_{0}\right) \geq G\left(z_{1}, \gamma_{0}\right)-N\left(\gamma_{1}-\gamma_{0}\right) \\
& =G\left(z_{1}, \gamma_{0}\right)-G\left(z_{1}, \gamma_{1}\right)+G\left(z_{1}, \gamma_{1}\right)-N\left(\gamma_{1}-\gamma_{0}\right)
\end{aligned}
$$




$$
\geq-N\left(\gamma_{1}-\gamma_{0}\right)+G\left(z_{1}, \gamma_{1}\right)-N\left(\gamma_{1}-\gamma_{0}\right)=G\left(z_{1}, \gamma_{1}\right)
$$

Consequently, $\left(y_{1}, \lambda_{1}\right),\left(z_{1}, \gamma_{1}\right)$ are lower and upper solutions of problem (2).

Let us assume that

$$
\begin{gathered}
\lambda_{0} \leq \lambda_{1} \leq \ldots \leq \lambda_{k-1} \leq \lambda_{k} \leq \gamma_{k} \leq \gamma_{k-1} \leq \ldots \leq \gamma_{1} \leq \gamma_{0} \\
y_{0}(t) \leq y_{1}(t) \leq \ldots \leq y_{k-1}(t) \leq y_{k}(t) \leq z_{k}(t) \leq z_{k-1}(t) \leq \ldots \leq z_{1}(t) \leq z_{0}(t) \\
t \in J
\end{gathered}
$$

and

$$
\left\{\begin{array} { c } 
{ y _ { k } ^ { \prime } ( t ) \leq f ( t , y _ { k } ( t ) , \lambda _ { k } ) , \quad y _ { k } ( 0 ) = k _ { 0 } , } \\
{ 0 \leq G ( y _ { k } , \lambda _ { k } ) , }
\end{array} \quad \left\{\begin{array}{c}
z_{k}^{\prime}(t) \geq f\left(t, z_{k}(t), \gamma_{k}\right), \quad z_{k}(0)=k_{0} \\
0 \geq G\left(z_{k}, \gamma_{k}\right)
\end{array}\right.\right.
$$

for some $k>1$. We shall prove that:

$$
\left\{\begin{array}{c}
\lambda_{k} \leq \lambda_{k+1} \leq \gamma_{k+1} \leq \gamma_{k} \\
y_{k}(t) \leq y_{k+1}(t) \leq z_{k+1}(t) \leq z_{k}(t), \quad t \in J
\end{array}\right.
$$

and

$$
\begin{gathered}
\left\{\begin{array}{c}
y_{k+1}^{\prime}(t) \leq f\left(t, y_{k+1}(t), \lambda_{k+1}\right), \quad y_{k+1}(0)=k_{0} \\
0 \leq G\left(y_{k+1}, \lambda_{k+1}\right)
\end{array}\right. \\
\left\{\begin{array}{c}
z_{k+1}^{\prime}(t) \geq f\left(t, z_{k+1}(t), \gamma_{k+1}\right), \quad z_{k+1}(0)=k_{0} \\
0 \geq G\left(z_{k+1}, \gamma_{k+1}\right)
\end{array}\right.
\end{gathered}
$$

where

$$
\begin{gathered}
\left\{\begin{array}{c}
y_{k+1}^{\prime}(t)=f\left(t, y_{k}(t), \lambda_{k}\right), \quad y_{k+1}(0)=k_{0} \\
0=G\left(y_{k}, \lambda_{k}\right)-N\left(\lambda_{k+1}-\lambda_{k}\right),
\end{array}\right. \\
\left\{\begin{array}{c}
z_{k+1}^{\prime}(t)=f\left(t, z_{k}(t), \gamma_{k}\right), \quad z_{k+1}(0)=k_{0} \\
0=G\left(z_{k}, \gamma_{k}\right)-N\left(\gamma_{k+1}-\gamma_{k}\right) .
\end{array}\right.
\end{gathered}
$$

Put $p=\lambda_{k}-\lambda_{k+1}$, so:

$$
0=G\left(y_{k}, \lambda_{k}\right)-N\left(\lambda_{k+1}-\lambda_{k}\right) \geq-N\left(\lambda_{k+1}-\lambda_{k}\right)=N p
$$

and hence $\lambda_{k} \leq \lambda_{k+1}$. Let $p=\lambda_{k+1}-\gamma_{k+1}$. In view of $3^{\circ}$ and $4^{\circ}$, we see that:

$$
\begin{gathered}
0=G\left(y_{k}, \lambda_{k}\right)-N\left(\lambda_{k+1}-\lambda_{k}\right) \\
=G\left(y_{k}, \lambda_{k}\right)-G\left(z_{k}, \gamma_{k}\right)-N\left(\lambda_{k+1}-\lambda_{k}\right)+N\left(\gamma_{k+1}, \gamma_{k}\right) \\
\leq G\left(z_{k}, \lambda_{k}\right)-G\left(z_{k}, \gamma_{k}\right)-N\left(\lambda_{k+1}-\lambda_{k}\right)+N\left(\gamma_{k+1}-\gamma_{k}\right) \\
\leq N\left(\gamma_{k}-\lambda_{k}\right)-N\left(\lambda_{k+1}-\lambda_{k}\right)+N\left(\gamma_{k+1}-\gamma_{k}\right)=-N p .
\end{gathered}
$$


Hence we have $\lambda_{k+1} \leq \gamma_{k+1}$. Now, let $p=\gamma_{k+1}-\gamma_{k}$. Then:

$$
0=G\left(z_{k}, \gamma_{k}\right)-N\left(\gamma_{k+1}-\gamma_{k}\right) \leq-N p
$$

so $\gamma_{k+1} \leq \gamma_{k}$, which shows that the first inequality of (4) is satisfied.

As before, we set $p(t)=y_{k}(t)-y_{k+1}(t), t \in J$. Then:

$$
p^{\prime}(t)=y_{k}^{\prime}(t)-y_{k+1}^{\prime}(t) \leq f\left(t, y_{k}(t), \lambda_{k}\right)-f\left(t, y_{k}(t), \lambda_{k}\right)=0
$$

and $p(0)=0$, so $y_{k}(t) \leq y_{k+1}(t), t \in J$. We observe that for $p(t)=y_{k+1}(t)-$ $z_{k+1}(t), t \in J$, we have

$$
\begin{gathered}
p^{\prime}(t)=y_{k+1}^{\prime}(t)-z_{k+1}^{\prime}(t)-f\left(t, y_{k}(t), \lambda_{k}\right)-f\left(t, z_{k}(t), \gamma_{k}\right) \\
\leq f\left(t, z_{k}(t), \gamma_{k}\right)-f\left(t, z_{k}(t), \gamma_{k}\right)=0
\end{gathered}
$$

which proves that $y_{k+1}(t) \leq z_{k+1}(t), t \in J$. Put $p(t)=z_{k+1}(t)-z_{k}(t), t \in J$. Then we have:

$$
p^{\prime}(t)=z_{k+1}^{\prime}(t)-z_{k}^{\prime}(t) \leq f\left(t, z_{k}(t), \gamma_{k}\right)-f\left(t, z_{k}(t), \gamma_{k}\right)=0
$$

so $z_{k+1}(t) \leq z_{k}(t), t \in J$. Therefore:

$$
y_{k}(t) \leq y_{k+1}(t) \leq z_{k+1}(t) \leq z_{k}(t), \quad t \in J .
$$

It is simple to show that $\left(y_{k+1}, \lambda_{k+1}\right),\left(z_{k+1}, \gamma_{k+1}\right)$ are lower and upper solutions of problem (2).

Hence, by induction, we have:

$$
\begin{gathered}
\lambda_{0} \leq \lambda_{1} \leq \ldots \leq \lambda_{n} \leq \gamma_{n} \leq \ldots \leq \gamma_{1} \leq \gamma_{0} \\
y_{0}(t) \leq y_{1}(t) \leq \ldots \leq y_{n}(t) \leq z_{n}(t) \leq \ldots \leq z_{1}(t) \leq z_{0}(t), t \in J
\end{gathered}
$$

for all $n$. Employing standard techniques [4], it can be shown that the sequences $\left\{y_{n}, \lambda_{n}\right\},\left\{z_{n}, \gamma_{n}\right\}$ converge uniformly and monotonically to $(y, \lambda),(z, \gamma)$, respectively. Indeed, $(y, \lambda)$ and $(z, \gamma)$ are solutions of problem (2) in view of the continuity of $f$ and $G$, and the definitions of the above sequences.

We have to show that if $(u, \beta)$ is any solution of problem $(2)$ such that:

$$
y_{0}(t) \leq u(t) \leq z_{0}(t), t \in J, \text { and } \lambda_{0} \leq \beta \leq \gamma_{0},
$$

then:

$$
y_{0}(t) \leq y(t) \leq u(t) \leq z(t) \leq z_{0}(t), t \in J, \text { and } \lambda_{0} \leq \lambda \leq \beta \leq \gamma \leq \gamma_{0} .
$$

To show this, we suppose that:

$$
y_{k}(t) \leq u(t) \leq z_{k}(t), t \in J, \text { and } \lambda_{k} \leq \beta \leq \gamma_{k}
$$

for some $k$. Put $\beta=\lambda_{k+1}-\beta$. Then, in view of $3^{\circ}$ and $4^{\circ}$, we have

$$
\begin{gathered}
0=G\left(y_{k}, \lambda_{k}\right)-N\left(\lambda_{k+1}-\lambda_{k}\right) \leq G\left(u, \lambda_{k}\right)-N\left(\lambda_{k+1}-\lambda_{k}\right) \\
=G\left(u, \lambda_{k}\right)-G(u, \beta)-N\left(\lambda_{k+1}-\lambda_{k}\right) \\
\leq N\left(\beta-\lambda_{k}\right)-N\left(\lambda_{k+1}-\lambda_{k}\right)=-N p,
\end{gathered}
$$

so $p \leq 0$, and hence $\lambda_{k+1} \leq \beta$. Let $p=\beta-\gamma_{k+1}$. Then we obtain:

$$
0=G(u, \beta) \leq G\left(z_{k}, \beta\right)=G\left(z_{k}, \beta\right)-G\left(z_{k}, \gamma_{k}\right)+N\left(\gamma_{k+1}-\gamma_{k}\right)
$$




$$
\leq N\left(\gamma_{k}-\beta\right)+N\left(\gamma_{k+1}-\gamma_{k}\right)=-N p,
$$

and hence $p \leq 0$, so $\beta \leq \gamma_{k+1}$. This shows that:

$$
\lambda_{k+1} \leq \beta \leq \gamma_{k+1} \text {. }
$$

As before, we set $p(t)=y_{k+1}(t)-u(t), t \in J$. In view of $2^{\circ}$, we obtain:

$$
\begin{gathered}
p^{\prime}(t)=y_{k+1}^{\prime}-u^{\prime}(t)=f\left(, y_{k}(t), \lambda_{k}\right)-f(t, u(t), \beta) \\
\leq f(t, u(t), \beta)-f(t, u(t), \beta)=0 ;
\end{gathered}
$$

hence $p(t) \leq 0, t \in J$, and $y_{k+1}(t) \leq u(t), t \in J$. Now let $p(t)=u(t)-z_{k+1}(t)$, $t \in J$. We see that:

$$
\begin{gathered}
p^{\prime}(t)=u^{\prime}(t)-z_{k+1}^{\prime}(t)=f(t, u(t), \beta)-f\left(t, z_{k}(t), \gamma_{k}\right) \\
\leq f\left(t, z_{k}(t), \gamma_{k}\right)-f\left(t, z_{k}(t), \gamma_{k}\right)=0
\end{gathered}
$$

and $p(t) \leq 0, t \in J$, so $u(t) \leq z_{k+1}(t), t \in J$. This shows that:

$$
y_{k+1}(t) \leq u(t) \leq z_{k+1}(t), t \in J
$$

By induction, this proves that the inequalities:

$$
y_{n}(t) \leq u(t) \leq z_{n}(t), \quad t \in J, \text { and } \lambda_{n} \leq \beta \leq \gamma_{n}
$$

are satisfied for all $n$. Taking the limit as $n \rightarrow \infty$, we conclude that:

$$
y(t) \leq u(t) \leq z(t), t \in J, \text { and } \lambda \leq \beta \leq \gamma .
$$

Therefore, $(y, \lambda),(z, \gamma)$ are minimal and maximal solutions of (2). The proof is complete.

\section{References}

[1] Goma, A., The method of successive approximations in a two-point boundary value problem with a parameter, Ukr. Mat. Zh. 29 (1977), 800-807. (In Russian)

[2] Jankowski, T., Existence, uniqueness and approximate solutions of problems with a parameter, Zesz. Nauk. Politech. Gdansk Mat. 16 (1993), 3-167.

[3] Kurpiel, N.S. and Marusiak, A.G., A multipoint boundary value problem for differential equations with parameters, Ukr. Mat. Zh. 32 (1980), 223-226. (In Russian)

[4] Ladde, G.S., Lakshmikantham, V. and Vatsala, A.S., Monotone Iterative Techniques for Nonlinear Differential Equations, Pitman, Boston 1985.

[5] Pomentale, T., A constructive theorem of existence and uniqueness for the problem $y^{\prime}=f(x, y, \lambda), y(\alpha), \alpha, y(b)=\beta, Z$. Angew. Math. Mech. 56 (1976), 387-388. 


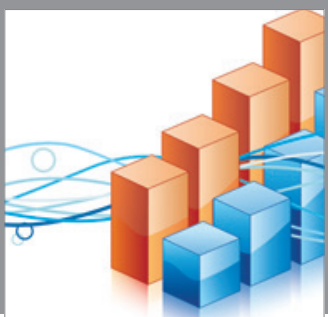

Advances in

Operations Research



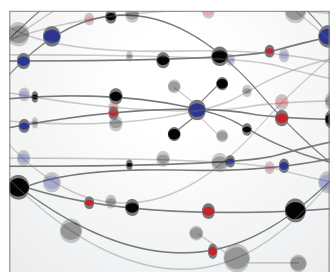

\section{The Scientific} World Journal
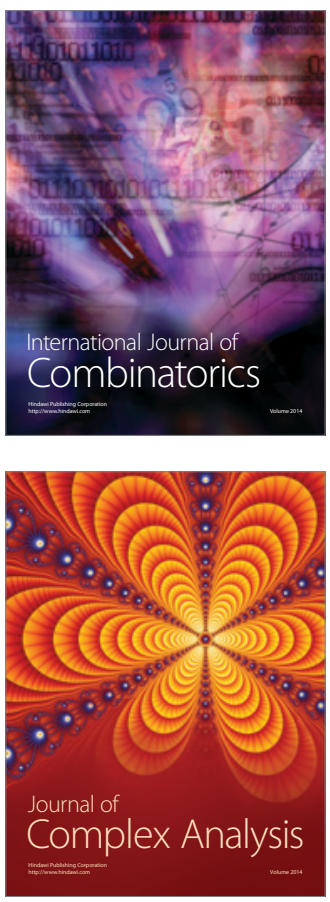

International Journal of

Mathematics and

Mathematical

Sciences
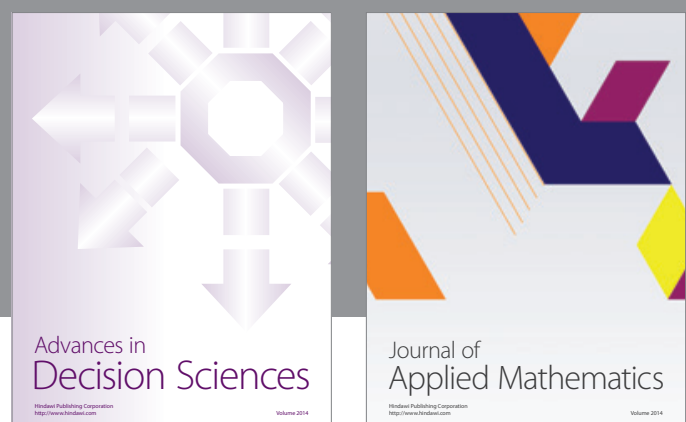

Journal of

Applied Mathematics
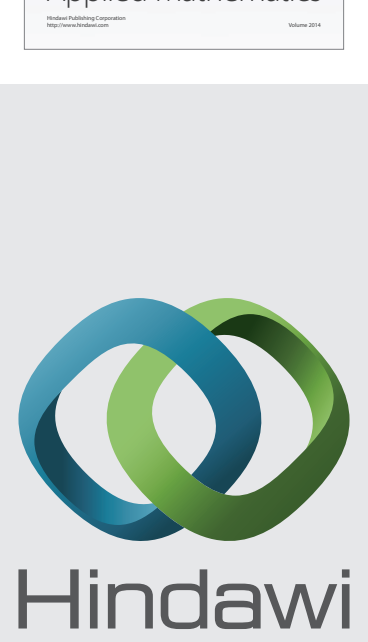

Submit your manuscripts at http://www.hindawi.com
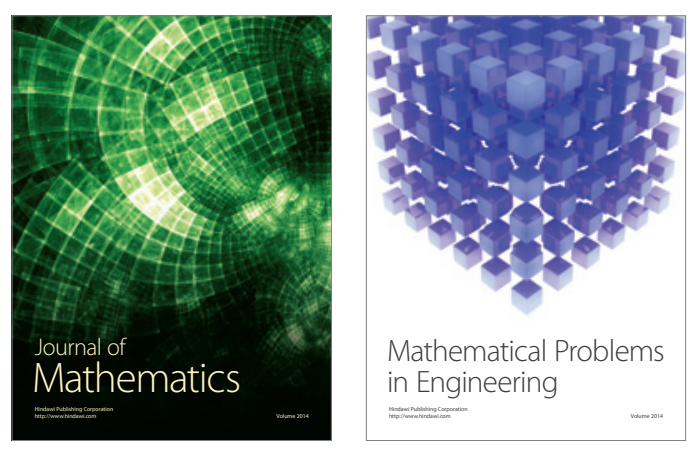

Mathematical Problems in Engineering
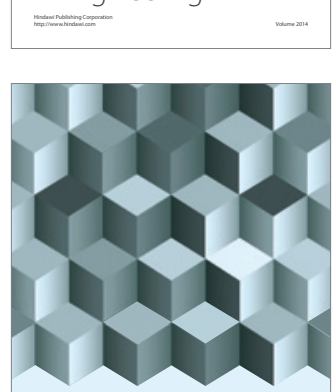

Journal of

Function Spaces
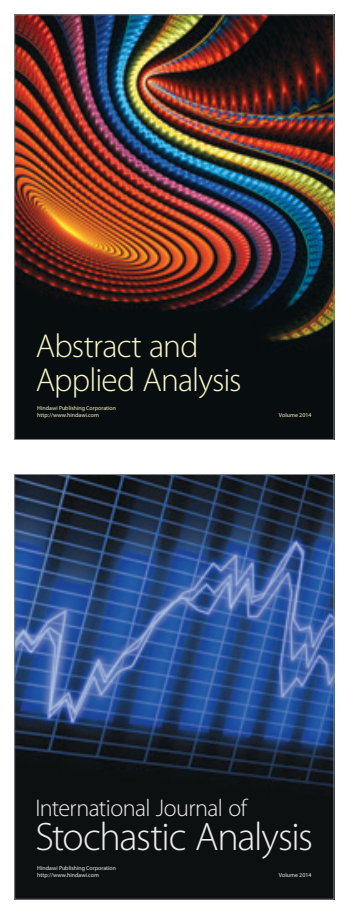

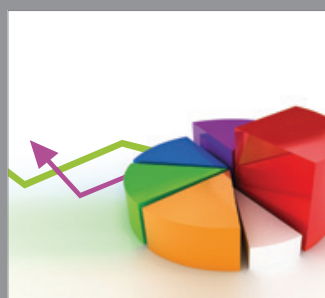

ournal of

Probability and Statistics

Promensencen
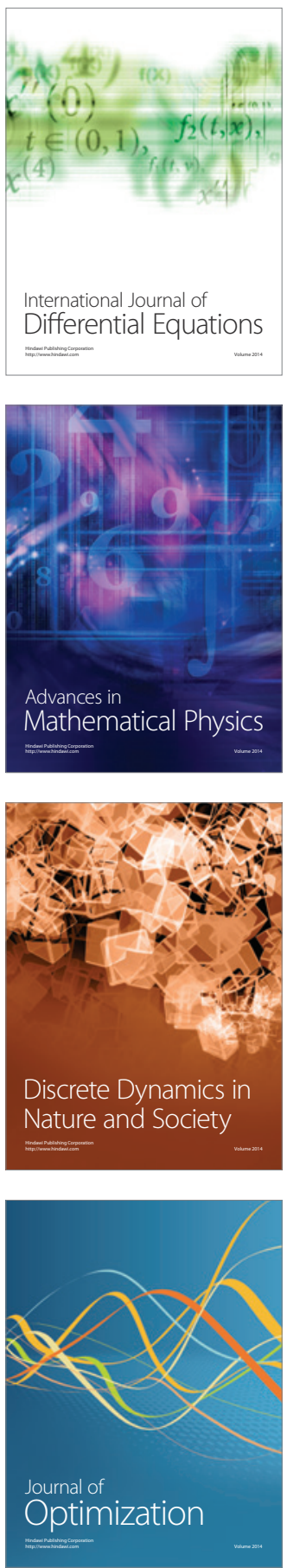